\title{
BMJ Open Work-related stressors among hospital physicians: a qualitative interview study in the Tokyo metropolitan area
}

\author{
Yuko Ihara (iD , ${ }^{1}$ Daisuke Son (D) , ${ }^{2}$ Masahiro Nochi (D) , ${ }^{1}$ Ryu Takizawa (iD ${ }^{1,3,4}$
}

To cite: Ihara Y, Son D, Nochi M, et al. Work-related stressors among hospital physicians: a qualitative interview study in the Tokyo metropolitan area. BMJ Open 2020;10:e034848. doi:10.1136/ bmjopen-2019-034848

- Prepublication history for this paper is available online. To view these files, please visit the journal online (http://dx.doi org/10.1136/bmjopen-2019034848).

Received 08 0ctober 2019 Revised 17 August 2020 Accepted 19 August 2020
Check for updates

(C) Author(s) (or their employer(s)) 2020. Re-use permitted under CC BY-NC. No commercial re-use. See rights and permissions. Published by BMJ.

For numbered affiliations see end of article.

\section{Correspondence to}

Yuko Ihara, Clinical Psychology, Integrated Educational Sciences, Graduate School of Education, The University of Tokyo, 7-31, Hongo, Bunkyo-ku, Tokyo, Japan;

yuko_ihara@icloud.com and

Dr Ryu Takizawa;

takizawar-tky@umin.ac.jp

\section{ABSTRACT}

Objectives We explored Japanese physicians' workrelated stressors and identified those unique to this population, as well as clarified the influence of cultural and medical system diversity on these stressors to determine the content of future stress-reducing interventions for hospital physicians in Japan.

Design We conducted a semistructured, face-to-face interview-based qualitative study between August and October 2017. The collected data were analysed using the grounded theory approach.

Setting Hospitals around the Tokyo metropolitan area, Japan.

Participants Sixteen hospital physicians (mean age $(S D)=33.9$ (4.2) years; 11 men, 5 women). Seven worked in internal medicine and nine in surgery.

Results We found unique stressors related to the Japanese medical system and culture, such as continuous all-day work after night shifts, and a hierarchical organisational system called lkyoku. The results also indicated that Japanese physicians shared several stressors with Western physicians, such as sleep deprivation, high pressure and the limits of medicine. Conclusions Our study clarifies some sources of workrelated stressors among hospital physicians in Japan. While the key components of Western interventions might be useful in a Japanese context, the original evidence obtained from this study highlights the necessity of initiating interventions addressing the unique stressors of Japanese physicians. To reduce physicians' stress and enhance their well-being, psychological interventions for hospital physicians must be introduced in Japan.

\section{INTRODUCTION}

Physician burnout is a major problem in many countries, given its serious negative effects on patient care and physicians' own career satisfaction. ${ }^{1-3}$ Burnout is a psychological syndrome comprising emotional exhaustion, depersonalisation and reduced personal accomplishment. ${ }^{4}$ The US national studies showed more than $50 \%$ of the physicians reported at least one symptom of burnout. ${ }^{5}$ The study reported that burned out physician had 5\%-11\% increasing likelihood of reporting a major medical error. ${ }^{6}$ Physician burnout is the consequence of accumulated

\section{Strengths and limitations of this study}

This is the first qualitative study that explored essential information on work-related stressors for Japanese hospital physicians.

- The sample size was small despite including diverse specialists because recruitment was challenging.

- However, the use of face-to-face interviews helped create an in-depth exploration of how diversity in the cultural and medical system influences workrelated stressors for Japanese physicians.

stress in the workplace. ${ }^{78}$ The National Institute for Occupational Safety and Health defines job stress as 'the harmful physical and emotional responses that occur when the requirements of the job do not match the capabilities, resources, or needs of the worker'. 9

Physician occupational stress has been extensively studied in Western countries, particularly the USA, the UK and Canada. Studies have suggested that the leading causes of resident physicians' stress and impairment formed three major categories: situational, personal and professional stressors. ${ }^{10}{ }^{11}$ However, as our focus was not only on resident physicians, and Western literature has widely researched physicians' stressors, we found that there are actually four categories of physicians' stressors, as follows: (1) situational stressors including sleep deprivation, ${ }^{12} 13$ heavy workload, ${ }^{1415}$ work conditions (eg, 'dealing with death'12 and 'long working hours and irregular work schedules, ${ }^{16}$ ), time pressure, ${ }^{17-20}$ lack of resources ${ }^{21} 22$ and position; ${ }^{12}$ (2) personal stressors including a lack of personal and/or family life, ${ }^{17} 1920$ relocation, ${ }^{14}$ lack of social support, ${ }^{18} 23$ financial matters ${ }^{24}$ psychological conflict (eg, 'personal morals and ethical dilemmas involved in patient treatment' and 'performing an abortion'13) and personality traits (eg, 'doctors who are most stressed showed higher levels of neuroticism'); $;^{25}$ (3) professional stressors 
including interpersonal conflict with coworkers, ${ }^{12}{ }^{23}$ interpersonal conflict with patients and their families, ${ }^{1727}$ lack of autonomy (eg, 'job constraints ${ }^{28}$ and 'the limits of medical treatments ${ }^{15} 29$ ), academic pressure,${ }^{17} 20$ paperwork, ${ }^{16}$ high level of responsibility, ${ }^{12}$ social pressure, ${ }^{27}$ medical errors and litigation, ${ }^{1823}$ patient care (eg, 'coping with dying patients, 28 ), need for continuous study, ${ }^{15} 28$ challenges of electronic health records ${ }^{30}$ and uncertain future ${ }^{14}$ and (4) demographic stressors including age, ${ }^{26} 31$ sex, ${ }^{20}{ }^{23}$ marital status, ${ }^{21}$ educational background ${ }^{32}$ and specialisation. $^{21} 33$

To cope with these stressors, with the emergence of physician health programmes, numerous psychological interventions are being implemented in Western countries, including psychological education workshops, peer support and counselling and so on ${ }^{34}$ Such support strategies were exclusively developed for physicians, who have specific needs related to confidentiality and stigma. ${ }^{35}$ Furthermore, Western countries implemented these strategies for both protecting physicians' well-being and enhancing the quality of patient care.

In Japan, actions targeting patient safety are being taken, such as establishing a reporting system within the hospital or holding training seminars on patient safety for hospital staff. ${ }^{36}$ However, these measures attempt to prevent medical errors by increasing the attention levels of healthcare providers. Studies showed that the prevalence of burnout among Japanese physician is over $20 \%-40 \%,{ }^{3738}$ and there are no concrete psychological attempts aimed at protecting physicians' health. Even the work hour regulations for physicians were not strictly established in Japanese hospitals until only recently, when the Japanese government announced an action plan for work reforms that included the implementation of overtime management strategies for all physicians by $2024 .{ }^{39}$ This action plan finally emphasised the importance of preserving physicians' mental health and the necessity of psychological intervention.

To develop psychological interventions for physicians in Japan, it is first necessary to consider whether Western approaches are appropriate for Japanese physicians. Importantly, the Japanese physician culture and medical system differ from those in Western countries. For example, recently, the share of female physicians has been increasing in all Organisation for Economic Co-operation and Development (OECD) countries. However, the share of female physicians in Japan is only $20 \%$, the lowest share among 34 OECD member countries. ${ }^{40}$ The Japanese government reported detailed rates of female physician in hospitals by specialty: $17.2 \%$ internists and $7.8 \%$ surgeons. $^{41}$

Another distinctive difference in Japanese hospital physicians is the employment system called Ikyoku. Ikyoku is a Japanese organisational system for physicians who are finishing their first 2 years of clinical internship which began in the 1870 s. $^{42}{ }^{43}$ Each clinical department within the university hospital has an Ikyoku, and it functions somewhat like an independent organisation. Its role

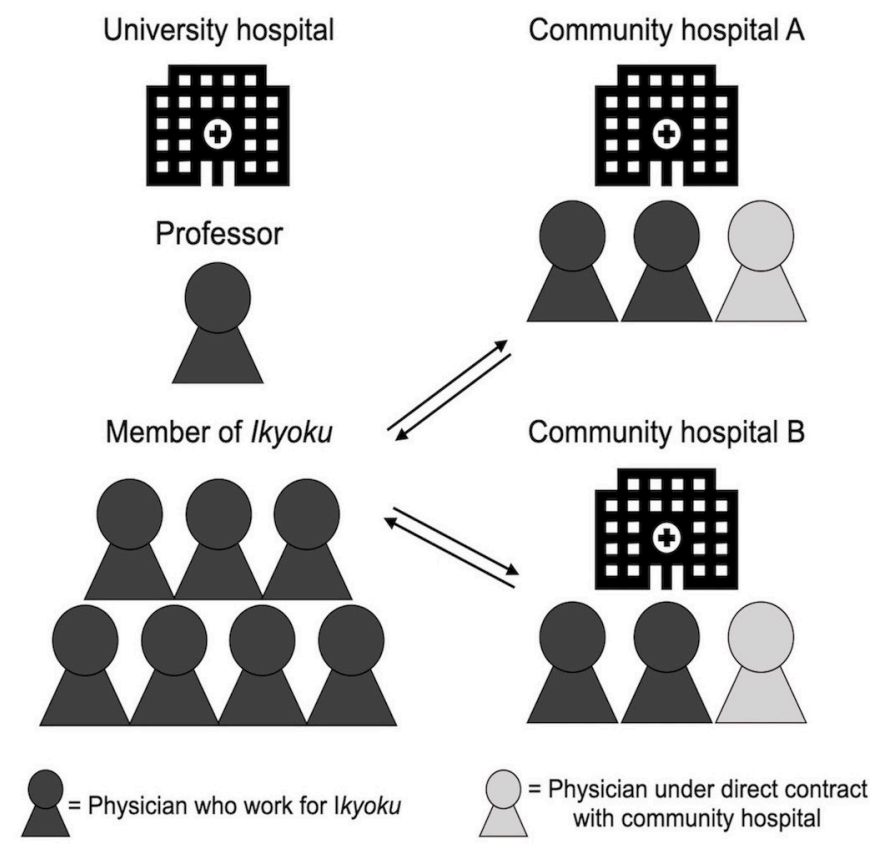

Figure 1 Hiring process of Japanese hospital.

is to educate and support the practice and research of undergraduate/postgraduate physicians. Furthermore, the Ikyoku has developed into a pool of physicians that can be assigned to affiliated community hospitals. ${ }^{44}$ The chiefs of Ikyoku are professors in the clinical department, and they have the authority to manage personnel affairs. Physicians who work for Ikyoku go back and forth between university and community hospitals every few months for several years regardless their intention (see figure 1 ). Their salary is fixed in the contract with the hospital in which they work. Generally, community hospital salaries are higher than university hospital salaries. That is to say, the place of work and income of Ikyoku physicians are not stable. However, more than $70 \%$ of the hospital physicians in Japan who have finished the 2-year internship after graduated medical school work under the Ikyoku system with no direct contract with a community hospital. ${ }^{45}$

These cultural and systematic differences may reflect on work-related stressors; thus, interventions tailored for Japanese physicians should be considered. Contrary to those of Western physicians, the stressors affecting Japanese physicians have barely been studied. Existing studies have typically used quantitative methodologies, evaluating physicians' stress levels and stressors using questionnaires developed for non-medical personnel. They also often grouped physicians with other healthcare providers. However, there is not yet enough empirical research to reveal the kind of stress that Japanese physicians experience related to their work.

Therefore, our objective was to examine Japanese hospital physicians' stressors to determine the features of psychological interventions tailored for Japanese physicians. The main aim of this study was to explore Japanese hospital physicians' work-related stressors. A secondary aim was to clarify the influence of cultural and medical 
system diversity on the unique stressors of Japanese physicians and determine whether psychological interventions for Japanese physicians should differ from those designed for Westerners.

\section{METHODS}

\section{Participants and recruitment}

The inclusion criteria for the study included physicians with 3-15 years of experience as a practitioner (ie, finishing 2 years of internship and having a licence to practice medicine independently in Japan) who worked in a hospital with over 400 beds in the Tokyo metropolitan area. In a previous survey conducted by the Japanese Medical Association, physicians working in hospitals with a large number of beds and less experienced physicians were considered to be at a high risk for stress. ${ }^{46}$ Additionally, medical interns with under 2 years' experience were excluded because Japanese interns do not work in specific clinical departments, instead, their clinical training location changes every $1-3$ months.

Recruitment was challenging because the physicians who met the above criteria were all remarkably busy. We intended to find participants who, despite their full schedules, could find time to participate; therefore, a recruitment sheet was sent via e-mail or social networking sites to 31 physicians (20 physicians who were personal or collegial contacts of the researchers; 11 were recruited by snowball sampling method). The recruitment sheet contained detailed information on the study's aims and explained that participation would require talking about psychological work stress. Seventeen physicians agreed to participate; the others either gave no response or refused, primarily because of their busy work schedules. One physician, who did not satisfy our recruitment criteria at the time of the interview, was later excluded. The search for new participants concluded when new subcategories no longer appeared, even though the detailed descriptions were sometimes different.

Sixteen hospital physicians (11 men, 5 women) participated in this study. Their age ranged from 26 to 40 years old (mean (SD)=33.9 (4.2) years). Their experience working in clinical practice ranged from 3 to 14 years, including two resident years (mean $(\mathrm{SD})=9.4$ (3.8) years). One allergy and rheumatology physicians, three cardiologists, three digestive surgeons, one emergency physician, one geriatrician, one nephrology and endocrinology physician, one neurologist, one neurosurgeon, three obstetrics and gynaecologists and one plastic surgeon participated. Also, $87.5 \%$ of the physicians were part of an Ikyoku, nearly $70 \%$ worked at university hospitals at the time of the study and the rest had been working at community hospitals.

\section{Data collection methods}

A semistructured, individual interview method was used to collect all data. The duration of the interview varied from 10 to $42 \mathrm{~min}$ (20 min on average). Interviews were conducted between August and October 2017, in person, and at a location chosen by the participant, which usually consisted of their offices or hospital coffee shops. All interviews were conducted by one researcher (trainee clinical psychologist at the period of the study), digitally recorded and later transcribed by the interviewer. All interviews were conducted using an interview schedule (see online supplemental file) and focused on physicians' work-related stress. The interview schedule was developed following a review of Western literature: backup prompts referring Western physicians' stressors were made in case participants were unable to sufficiently discuss their stress.

\section{Patient and public involvement}

The patients and the public were not involved in this study.

\section{Data analysis}

The verbatim transcripts of the interviews were used for data analysis. Before analysis, all identifying information was removed from the data. The transcripts were closely read by all researchers. MAX QDA V.12, a qualitative data analysis software program, was then used to help with the coding and categorisation process. The data were analysed using the grounded theory approach, which is characterised by constant comparison between raw data and analysis results to ensure there is never a departure from the data. ${ }^{47}$

Four procedures were conducted to analyse the data (see box 1). On completing the coding process, the subcategories and categories were confirmed by the primary researcher and two graduate clinical psychology students with experience in qualitative data analysis. The code names were updated to increase inter-rater reliability before settling on the subcategorisation. In order to easily find unique stressors affecting Japanese physicians, we derived steps 3 and 4 . Only when the Japanese stressors were not similar to those of Westerners were the stressors considered unique.

\section{Box 1 Analytical data processing}

Step 1: Segmentation: wherein the transcript data were divided into segments of meaning.

Step 2: Coding: where a code name was provided to each segment that briefly summarises that segment.

Step 3: Subcategorisation: where similar codes are collected and given a subcategory name. The name of the subcategory was adjusted to fit the subcategories of stressors found for Western physicians in previous studies, when possible.

Step 4: Categorisation: wherein all subcategories were placed into a category corresponding to the stressors found among Western physicians (situational, personal, professional or demographic). When some subcategories were considered fitting into more than one category, we referred to the subcategories of Western physicians and put it into one category. 
Table 1 Japanese physicians' work-related stressors

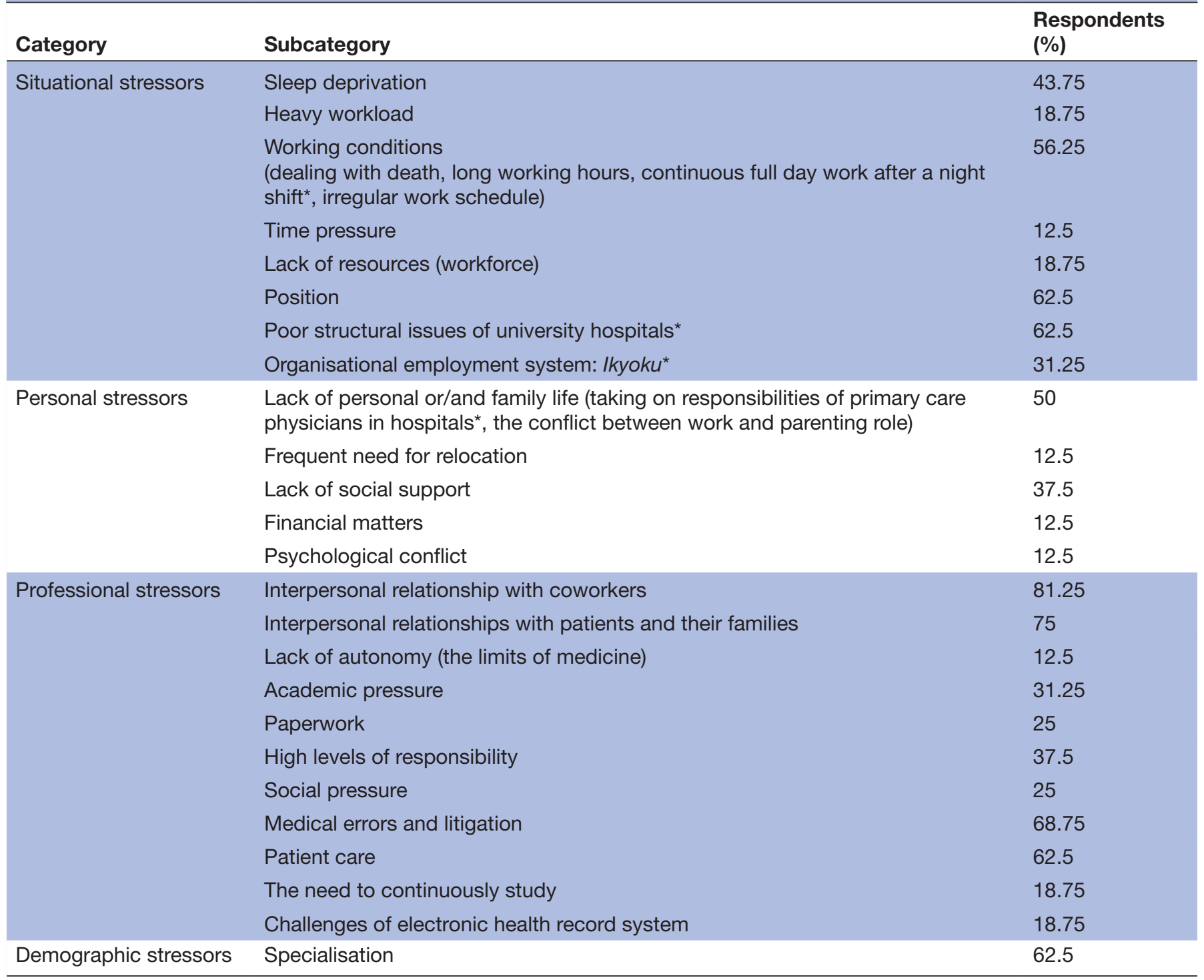

*Unique stressors for Japanese hospital physicians.

\section{FINDINGS}

Respondents mentioned multiple work-related stressors, which fit well within the four categories obtained in previous studies on Western physicians: situational, personal, professional and demographic stressors (see table 1).

\section{Situational stressors}

Situational stressors were related to work situation; for instance, work structure and regulation. The situational stressors found among Japanese physicians primarily corresponded to those of Westerners. Some working condition-related stressors were also found to be unique to Japanese physicians. Both Japanese and Western physicians were stressed because of their working conditions, including constant exposure to life-or-death situations, long working hours and irregular work schedules. However, the details of these stressors differed somewhat.
In Japan, there is no regulation restricting physicians' continuous working hours, so respondents often felt stressed about the need to engage in 'continuous full day work after a night shift'. While this stressor may seem similar to that of 'long working hours' identified in Western physicians, we considered this might be unique to Japanese physicians. A common representative quote on this stressor was as follows:

Even the next day, after finishing a night shift, I felt I had to work until late at night (at the hospital). I think that is the main reason I'm exhausted. I wish I could have a complete day off. Continuous work makes me feel tired and nauseous.

Other unique stressors mentioned by Japanese physicians were related to university hospitals. More than $90 \%$ of participants had experience working in both university 
and community hospitals, and they generally expressed dissatisfaction with the working conditions at university hospitals.

\section{'Poor structural issues of university hospitals'}

For example, participants complained about the poor quality of the night shift rooms for physicians (eg, no clean shower equipment, no towel rental and poor bed conditions), low salary and complicated workflow (eg, 'the decision-making process at the university hospitals took much longer than that at the community hospitals'). Some respondents also experienced stress due to the hospital allowing self-referral patients, as the following quotation illustrates:

People who use outpatient services (by self-referral) at university hospitals tend to be selective in various ways... I mean, they seem like troublesome cases. I do not mean that the disease condition or diagnosis is troublesome. Rather, they seem to be 'troublesome' people. ... This kind of person has excessively high expectations or does not want to change hospitals even when it is not necessary to treat them in the university hospital.

Additionally, more than half of the respondents mentioned feeling stressed when they were asked to perform activities that are not typically done by physicians at community hospitals, especially in university hospitals. While laws do determine the roles of each medical professional, tasks such as paperwork, transport of patients, blood sampling and injections are not clearly allocated. The following quote represents common responses on this situation:

I think this situation may be limited to university hospitals...there are many things that physicians have to do even though they are not necessarily done by physicians in other (community) hospitals...For example, when a doctor prescribes medication, and this medication is no longer necessary (because of the changes in the patient's status)...the doctor has to return the medication (to the pharmaceutical department). Another example concerns medical certification. Medical clerks have always made medical certification outlines in other hospitals...But in this hospital, until very recently, physicians had to write all the detailed information for certifications. I had to research all the medical records of the patient to fill in the blanks in their consultation history...in these examples, I think the doctor is doing too many things.

\section{'Organizational employment system: Ikyoku'}

Members of the Ikyoku have more opportunities for education, research and clinical practice than physicians who are not members ${ }^{43}$; however, many participants mentioned that these merits sometimes worked against them. The following quote represents common responses to this situation:

Young physicians who belong to the Ikyoku frequently change workplaces, every six months to a year, to gain experience on various cases. Even if I wished to settle down to work at a particular hospital, it would not be easy...my opinion would not be accepted (by the Ikyoku). There is also an option to find a job directly at the (community) hospital. But...if I do so, I would experience only similar cases (depending on the region or hospitals).

In the quotation above, the physician experienced stress related to the frequent need for relocation, which was a personal stressor. However, the respondent also experienced a dilemma in weighing the advantages and disadvantages of the Ikyoku.

\section{Personal stressors}

Personal stressors were issues relating to individual lifestyle pattern. The personal stressors affecting Japanese physicians were also similar to those of Westerners. While the lack of a personal and family life were stressors for both Japanese and Western physicians, the former explained that the primary source of stress was the difficulty in taking time off work because they had to assume the responsibilities of primary care physicians in hospitals.

\section{'Taking on responsibilities of primary care physicians in the hospitals'}

In Japan, a single physician takes charge of a patient's treatment until that patient is discharged. However, this system prevents hospital physicians from taking time off because they need to check their patients' conditions daily, even when another physician is on duty during off hours. Thus, Japanese physicians may experience the pressure of being responsible for their patients even after finishing their shifts. The following two quotes represents common responses to this situation:

I think an ideal working style during the weekends is that I'm in charge of patients instead of the other doctor on Saturday, then I take time off on Sunday. But there sometimes physicians will say Why are you not seeing the patient by yourself, when you have a responsibility to your patient?

My son is one year old, and only I was available to take care of him last Saturday. My patient's condition got worse, and an intern doctor called me. I couldn't go to the hospital because I couldn't take my eyes off my son. I had no choice but to give a directive to the intern by phone and e-mail. It was stressful. I think it would have been better if a duty doctor or another doctor in the hospital dealt with this kind of situation.

\section{Professional stressors}

Professional stressors refer to the stress mainly caused by the professional domain, such as patient care, emotional and career development of the physician. All of stressors 
found in this category were found in previous studies of Western physicians as well. For example, the high levels of responsibility (eg, If I fail in the surgery, my patient dies) and social pressure (eg, I feel that this profession is always demanded the image of an 'good person') (see table 1). No professional stressors were unique to Japanese physicians.

\section{Demographic stressors}

In the current qualitative study, many participants mentioned that stress resulted from the unique characteristics of their specialisation (eg, 'emergency doctors frequently experience violence from patients'). Japanese physicians did not mention stressors such as age, marital status, educational background and sex, which were factors for Western physicians. No stressors caused by demographic characteristics were unique to Japanese physicians.

\section{DISCUSSION}

This is the first qualitative study that explored the stressors among hospital physicians in Japan. Our participants stated that most of their stressors were similar to those reported by Western physicians in previous studies. Stressors unique to Japanese physicians were also mentioned, all of which seemed to be related to the Japanese organisational and medical culture. From the above, our findings provided new evidence documenting the need to introduce interventions for Japanese physicians according to their unique needs. Meanwhile, the information on whether personality traits, job constrain, uncertain future, age, sex, marital status and educational background were sources of stress, as found in Western quantitative studies, could not be obtained by this qualitative study. However, since there were common stressors among Japanese and Western physicians, Western strategies for dealing with physicians' health may be also useful for Japanese physicians, such as a working time restriction directive and psychological intervention. ${ }^{34} 48$

Some unique stressors among Japanese physicians, such as continuous full days of work even after a night shift and taking on the responsibilities of primary care physicians at the hospitals, were related to stress due to overwork. It is known that these types of stressors may cause physical and psychological problems called 'karoshi (overwork death)' or 'karojisatsu (commit suicide due to overwork)', which represents occupational threats to Japanese physicians. ${ }^{49}$ Thus, the working time restriction policy should be considered in Japan's legislation. Mainly in Europe, working time restrictions for physicians have been discussed since $1998{ }^{48}$ Since 2017 , the Japanese government has been planning to finally implement an overtime restriction for Japanese hospital physicians; however, a more detailed restriction for the interdiction of continuous all-day work after a night shift and establishing rest periods are needed. Stress related to 'poor structural issues of university hospitals' was a unique stressor of Japanese physicians. The system of university hospitals in Japan may be unique due to the existence of the Ikyoku system. Japanese university hospitals contain multiple clinical departments, but each clinical department has an independent management system. Therefore, there are no unified rules as in community hospitals. To deal with this problem, it is necessary to implement hospital organisational reforms, such as intensification of teamwork between multiple Ikyoku. The aforementioned legally and organisationally mandated changes to the hospitals' working conditions are essential, but they often take a substantial amount of time to take effect. In the short term, then, it is necessary to focus on individual well-being by implementing psychological intervention strategies.

Some stressors permeate all areas of the physicians' career for as long as it lasts, such as patient care, lack of personal or/and family life, interpersonal relationship with coworker and high levels of responsibility and so on. Psychological intervention may be needed for the physicians to handle and reduce these types of stressors and enhance their well-being. In Western countries, individual training or group workshops were provided with the goal of increasing resilience skills and reducing stress among physicians ${ }^{5051}$ Moreover, social activities including retreats, collegiality events and health-related activity programmes were implemented to maintain individual well-being and to increase the opportunities of expanding and/or maintaining social support. Furthermore, sometimes physicians' families could join these programmes in order to promote a work-family balance. ${ }^{52-54}$ Also, peer support groups, individual counselling and coaching sessions were helpful in relieving the common stressors among physicians (such as dealing with death, social pressure, high levels of responsibility and so on). ${ }^{55} 56$

These psychological interventions may also be useful for Japanese physicians. However, to introduce these types of psychological interventions, we should consider regarding the Japanese collectivistic cultural background. Unique stressors affecting Japanese physicians, such as 'organizational employment system: Ikyoku', may be related to the country's cultural background. In fact, multiple studies of Japanese modes of communication have revealed that the Japanese collectivistic culture prevents individuals to assert their own opinions, especially towards elders or authority figures in order to maintain harmony within a social group. ${ }^{57-59}$ Therefore, it might be better to divide groups by age, for instance, when considering psychological interventions. Also, strategies to learn and practice assertive communication during medical practice and in day-to-day conversations at the workplace may be needed among Japanese physicians.

Our qualitative interviews were optimal for understanding the current stressors of hospital physicians in Japan, which had not been explored previously. However, this study presents a few limitations. First, there was a self-selection bias. The participants were recruited based on our personal knowledge or the recommendation of 
others and the sample consisted of individuals who had time/interest on this study. Our participants were mainly physicians who were part of an Ikyoku. It is likely that physicians who are directly employed by community hospitals or in private practice have other opinions. Additionally, the clinical departments of the participants varied considerably, and the characteristics of each department might have influenced work-related stress. The stressors could be different between our pool of participants, all working in the Tokyo metropolitan area, and physicians working in rural areas. Future studies should recruit a larger sample of physicians from multiple clinical departments and regions to confirm whether the stressors found in the current study are representative of Japanese hospital physicians in general. Second, our results are not strictly comparable with those of the studies surveying Westerners. All our information on Western physicians' stressors was obtained by reviewing several types of studies. Furthermore, Western physicians did not always have similar attributes, such as position or specialisation, as the physicians in our study. Accordingly, the stressors considered unique to Western physicians may not be applicable to Japanese physicians. Future studies should focus on Japanese physicians who work under similar conditions as those in previous Western studies to enable a more rigorous comparison.

\section{CONCLUSIONS}

This is the first empirical study to exploring Japanese hospital physicians' work-related stressors. Although Japanese physicians showed unique stressors, they share many commonalities with Western physicians. Future psychological support for Japanese physicians might use Western interventions to promote physicians' health. However, taking into consideration the unique stressors affecting Japanese physicians, which reflected the Japanese organisational and medical system and Japanese culture, might prove useful.

\section{Author affiliations \\ ${ }^{1}$ Clinical Psychology, Integrated Educational Sciences, Graduate School of Education, The University of Tokyo, Tokyo, Japan \\ ${ }^{2}$ Medical Education Studies, International Research Center for Medical Education, Graduate School of Medicine, The University of Tokyo, Tokyo, Japan \\ ${ }^{3}$ Neuropsychiatry, Graduate School of Medicine, The University of Tokyo, Tokyo, Japan \\ ${ }^{4}$ MRC Social, Genetic and Developmental Psychiatry Centre, Institute of Psychiatry, Psychology and Neuroscience, King's College London, London, United Kingdom}

Acknowledgements The authors would like to thank all the participants of this study. The authors would also like to thank H Shimoyama, H Kurihara, Y Nakashima, T Hosaka, K Naemura, M Eto and A Horita for their technical advice about the study design.

Contributors $\mathrm{YI}$ and RT designed this study. YI and DS recruited participants, and $\mathrm{YI}$ acquired data. All authors analysed and interpreted data. YI wrote the first draft of this manuscript, and DS, MN and RT revised the manuscript. All authors read and approved the final manuscript.

Funding This work was supported by The University of Tokyo Graduate School of Education International Academic Research Grant for Yl; The Royal Society and the British Academy grant number AL150003 and The Japanese Society for the Promotion of Science grant number JP16H05653 for RT.

Competing interests None declared.

Patient consent for publication Not required.

Ethics approval This study obtained ethical approval by the appropriate ethics review board. All participants signed an informed consent form.

Provenance and peer review Not commissioned; externally peer reviewed.

Data availability statement № additional data are available.

Open access This is an open access article distributed in accordance with the Creative Commons Attribution Non Commercial (CC BY-NC 4.0) license, which permits others to distribute, remix, adapt, build upon this work non-commercially, and license their derivative works on different terms, provided the original work is properly cited, appropriate credit is given, any changes made indicated, and the use is non-commercial. See: http://creativecommons.org/licenses/by-nc/4.0/.

ORCID iDs

Yuko Ihara http://orcid.org/0000-0002-1933-9982

Daisuke Son http://orcid.org/0000-0002-7053-162X

Masahiro Nochi http://orcid.org/0000-0002-0467-0883

Ryu Takizawa http://orcid.org/0000-0002-3876-0131

\section{REFERENCES}

1 Fahrenkopf AM, Sectish TC, Barger LK, et al. Rates of medication errors among depressed and burnt out residents: prospective cohort study. BMJ 2008;336:488-91.

2 Firth-Cozens J, Greenhalgh J. Doctors' perceptions of the links between stress and lowered clinical care. Soc Sci Med 1997;44:1017-22.

3 West CP, Dyrbye LN, Erwin PJ, et al. Interventions to prevent and reduce physician burnout: a systematic review and meta-analysis. Lancet 2016;388:2272-81.

4 Maslach C, Jackson SE, Leiter MP. Maslach burnout inventory manual. 192. 3rd ed. Palo Alto, CA: Consulting Psychologists Press, 1996.

5 Shanafelt TD, Hasan O, Dyrbye LN, et al. Changes in burnout and satisfaction with work-life balance in physicians and the general us working population between 2011 and 2014. Mayo Clin Proc 2015:90:1600-13.

6 Shanafelt TD, Balch CM, Bechamps G, et al. Burnout and medical errors among American surgeons. Ann Surg 2010;251:995-1000.

7 Linzer M, Poplau S, Babbott S, et al. Worklife and wellness in academic general internal medicine: results from a national survey. $J$ Gen Intern Med 2016;31:1004-10.

8 Buck K, Williamson M, Ogbeide S, et al. Family physician burnout and resilience: a cross-sectional analysis. Fam Med 2019;51:657-63.

9 National Institute for occupational safety and health. Stress at work. DHHS (NIOSH) Publication, 1999: 99-101. https://www.cdc.gov/ niosh/docs/99-101/

10 Aach RD, Conney T, Girard D, et al. Stress and impairment during residency training strategies for reduction, identification, and management. Ann Intern Med 1988;109:154-61.

11 Levey RE. Sources of stress for residents and recommendations for programs to assist them. Acad Med 2001;76:142-50.

12 Coomber S, Todd C, Park G, et al. Stress in UK intensive care unit doctors. Br J Anaesth 2002;89:873-81.

13 Power KG, Swanson V. Medical profession and stress. In: Encyclopaedia of stress. 2nd ed. San Diego, CA: Academic Press, 2007: 674-8.

14 Hayes B, Fitzgerald D, Doherty S, et al. Quality care, public perception and quick-fix service management: a Delphi study on stressors of hospital doctors in Ireland. BMJ Open 2015;5:e009564.

15 Richardsen AM, Burke RJ. Occupational stress and job satisfaction among physicians: sex differences. Soc Sci Med 1991;33:1179-87.

16 Cooper CL, Rout U, Faragher B. Mental health, job satisfaction, and job stress among general practitioners. BMJ 1989;298:366-70.

17 Agius RM, Blenkin H, Deary IJ, et al. Survey of perceived stress and work demands of consultant doctors. Occup Environ Med 1996;53:217-24.

18 Dunn PM, Arnetz BB, Christensen JF, et al. Meeting the imperative to improve physician well-being: assessment of an innovative program. $J$ Gen Intern Med 2007;22:1544-52.

19 Swanson V, Power KG, Simpson RJ. Occupational stress and family life: a comparison of male and female doctors. J Occup Organ Psychol 1998;71:237-60. 
20 Gender WJ. The work-life interface and wellbeing: a study of hospital doctors. Gend Work Organ 2013;20:439-53.

21 Shanafelt TD, Boone S, Tan L, et al. Burnout and satisfaction with work-life balance among US physicians relative to the general US population. Arch Intern Med 2012;172:1377-85.

22 Weigl M, Müller A, Zupanc A, et al. Hospital doctors' workflow interruptions and activities: an observation study. BMJ Qual Saf $2011 ; 20: 491-7$

23 Firth-Cozens J. Individual and organizational predictors of depression in general practitioners. Br J Gen Pract 1998;48:1647-51.

24 Hayes B, Prihodova L, Walsh G, et al. What's up doc? a national cross-sectional study of psychological wellbeing of hospital doctors in Ireland. BMJ Open 2017;7:e018023.

25 Christensen JF, Levinson W, Dunn PM. The heart of darkness: the impact of perceived mistakes on physicians. J Gen Intern Med 1992;7:424-31.

26 McManus IC, Keeling A, Paice E. Stress, burnout and doctors' attitudes to work are determined by personality and learning style: a twelve year longitudinal study of UK medical graduates. BMC Med 2004;2:29.

27 Pedrazza M, Berlanda S, Trifiletti E, et al. Exploring physicians' dissatisfaction and work-related stress: development of the PhyDis scale. Front Psychol 2016;7:1238.

28 Tattersall AJ, Bennett P, Pugh S. Stress and coping in hospital doctors. Stress Med 1999;15:109-13.

29 Mawardi BH, Satisfactions MBH. Satisfactions, dissatisfactions, and causes of stress in medical practice. JAMA 1979;241:1483.

30 Babbott S, Manwell LB, Brown R, et al. Electronic medical records and physician stress in primary care: results from the MEMO study. $J$ Am Med Inform Assoc 2014;21:e100-6.

31 Peisah C, Latif E, Wilhelm K, et al. Secrets to psychological success: why older doctors might have lower psychological distress and burnout than younger doctors. Aging Ment Health 2009;13:300-7.

32 Miller NM, McGowen RK. The painful truth: physicians are not invincible. South Med J 2000;93:966-73.

33 Tanner G, Bamberg E, Kozak A, et al. Hospital physicians' work stressors in different medical Specialities: a statistical group comparison. J Occup Med Toxicol 2015;10:7.

34 Braquehais MD, Tresidder A, DuPont RL. Service provision to physicians with mental health and addiction problems. Curr Opin Psychiatry 2015;28:324-9.

35 Brooks E, Gundersen DC, Gendel MH. Investing in physicians is investing in patients: enhancing patient safety through physician health and well-being research. J Patient Saf 2017;1.

36 Hirose M, Kawamura T, Igawa M, et al. Patient safety activity under the social insurance medical fee schedule in Japan: an overview of the 2010 nationwide survey. J Patient Saf 2017:1:PTS.0000000000000432.

37 Nishimura K, Nakamura F, Takegami M, et al. Cross-Sectional survey of workload and burnout among Japanese physicians working in stroke care: the nationwide survey of acute stroke care capacity for proper designation of comprehensive stroke center in Japan (JASPECT) study. Circ Cardiovasc Qual Outcomes 2014;7:414-22.

38 Saijo Y, Yoshioka E, Hanley SJB, et al. Job stress factors affect workplace resignation and burnout among Japanese rural physicians. Tohoku J Exp Med 2018;245:167-77.

39 Japanese Ministry of Health, Labour and Welfare. Work-style reform for doctors [lshi no hataraki kata kaikaku], 2017. Available: https:// www.mhlw.go.jp/file/05-Shingikai-12601000-SeisakutoukatsukanSanjikanshitsu_Shakaihoshoutantou/0000184303.pdf [Accessed 30 Sep 2019].

40 OECD. Women make up most of the health sector workers but they are under-represented in high-skilled jobs, 2017. Available: https:// www.oecd.org/gender/data/women-make-up-most-of-the-healthsector-workers-but-they-are-under-represented-in-high-skilled-jobs. htm [Accessed 27 fev 2020].
41 Japanese Ministry of Health, Labour and Welfare. Achievements of career support promotion project for female doctor and future initiatives [Josei-ishi career shien model hukyu suishin jigyo no seika to kongo no torikumi ni tsuite], 2018. Available: https://www.mhlw.go. jp/file/05-Shingikai-10801000-Iselkyoku-Soumuka/0000197435.pdf [Accessed 13 fev 2020]

42 Onishi H, Yoshida I. Rapid change in Japanese medical education. Med Teach 2004;26:403-8.

43 Otsuka T. The ikyoku system of university orthopedic surgery departments: an in-hospital organizational system unique to Japan-its significance for advancement of basic medical science related to orthopedic surgery. J Orthop Sci 2012;17:513-4.

44 Teo A. The current state of medical education in Japan: a system under reform. Med Educ 2007;41:302-8.

45 Japanese Ministry of Health, Labour and Welfare. Questionnaire survey on clinical training in 2017 [Heisei 29 nen rinshou kenshu shuryousha anqueto], 2017. Available: https://www.mhlw.go.jp/file/ 06-Seisakujouhou-10800000-Iselkyoku/0000182128.pdf [Accessed 27 fev 2020]

46 Japanese Medical Association. The report of project group to discuss work-style reform for doctors [Kinmui no kenkou shien ni kansuru project iinkai houkokusho], 2012. Available: http://dl.med.or. jp/dl-med/teireikaiken/20120328 6.pdf [Accessed 30 Sep 2019].

47 Glaser BG, Strauss AL. The discovery of grounded theory: strategies for qualitative research. Chicago, IL: Aldine Publishing Co, 1967.

48 Lambert TW, Smith F, Goldacre MJ. The impact of the European working time directive 10 years on: views of the UK medical graduates of 2002 surveyed in 2013-2014. JRSM Open 2016;7:205427041663270.

49 Hiyama T, Yoshihara M. New occupational threats to Japanese physicians: karoshi (death due to overwork) and karojisatsu (suicide due to overwork). Occup Environ Med 2008;65:428-9.

50 Sood A, Prasad K, Schroeder D, et al. Stress management and resilience training among department of medicine faculty: a pilot randomized clinical trial. J Gen Intern Med 2011;26:858-61.

51 Mache S, Vitzthum K, Klapp BF, et al. Evaluation of a multicomponent psychosocial skill training program for junior physicians in their first year at work: a pilot study. Fam Med 2015:47:693

52 Institute for Physician integration. The Institute for physician integration. Available: https://www.physicianintegration.org/ [Accessed 27 fev 2020].

53 Paolini HO, Bertram B, Hamilton T. Antidotes to burnout: fostering physician Resiliency, well-being, and holistic development. Medscape psychiatry, 2013. Available: https://www.medscape.com/ viewarticle/782514 [Accessed 27 fev 2020]

54 Stanford. Stanford health improvement program. Available: https:// hip.stanford.edu/programs/overview/ [Accessed 27 fev 2020].

55 Stanford Medicine. WellMD. Available: http://wellmd.stanford.edu/ [Accessed 27 fev 2020].

56 Gerada C, Warner L, Jones R, et al. The wounded healer: report on the first 10 years of practitioner health service. NHS practitioner health programme. Available: https://www.practitionerhealth.nhs. uk/media/content/files/PHP-report-web\%20version\%20final(1).pdf [Accessed 27 fev 2020].

57 Omura M, Stone TE, Levett-Jones T. Cultural factors influencing Japanese nurses' assertive communication. Part 1: Collectivism: Assertive communication and collectivism. Nurs Health $\mathrm{Sci}$ 2018;20:283-8

58 Omura M, Stone TE, Levett-Jones T. Cultural factors influencing Japanese nurses' assertive communication: Part 2 - hierarchy and power: Assertive communication and hierarchy. Nurs Health Sci 2018;20:289-95.

59 Singhal A, Nagao M. Assertiveness as communication competence a comparison of the communication styles of American and Japanese students. Asian J Commun 1993;3:1-18. 